\title{
AVALIAÇÃO DA SATISFAÇÃO SEXUAL EM UNIVERSITÁRIAS
}

\author{
Natália Trindade da Silva ${ }^{1}$, Silas de Oliveira Damasceno ${ }^{2}$. \\ ${ }^{1}$ Universidade Paulista - UNIP, Curso de Fisioterapia, Assis, SP. ${ }^{2}$ Universidade Estadual Paulista - UNESP, Residência \\ em Fisioterapia Aplicada à Neurologia, Presidente Prudente, SP. E-mail: nataliatrindades@gmail.com
}

\section{RESUMO}

A disfunção sexual é qualquer alteração no ciclo de resposta sexual, na qual, pode ser advinda de problemas psicológicos, biológicos ou sociais, por isso a necessidade de entendê-la. $\mathrm{O}$ objetivo do estudo foi avaliar a satisfação sexual em universitárias. A amostra foi composta por universitárias, com idades entre 18 e 35 anos, sexualmente ativas e que não realizaram tratamento de disfunção sexual recentemente. Foi aplicado o questionário Female Sexual Function Index (FSFI) que analisa seis domínios da função sexual: desejo, excitação, lubrificação, orgasmo, satisfação e dor e enfatiza o distúrbio da excitação, sendo $>27,5$ uma boa função sexual. A média geral obteve escore de 28,30 pontos, demonstrando boa função sexual e na média individual verificou-se que $28 \%$ das participantes sofrem de disfunção sexual. Conclui-se que as mulheres universitárias deste estudo apresentaram boa satisfação sexual, porém, com alta porcentagem de alguma disfunção sexual analisada pelo questionário FSFI.

Palavras-chave: satisfação sexual, disfunção sexual, questionário FSFI, fisioterapia.

\section{EVALUATION OF SEXUAL SATISFACTION IN UNIVERSITY WOMEN}

\section{ABSTRACT}

Sexual dysfunction is any change in the cycle of sexual response, in which, it may be due to psychological, biological or social problems, hence the need to understand it. The objective of the study was to evaluate sexual satisfaction in university women. The sample consisted of university women aged between 18 and 35 years who were sexually active and who had not recently been treated for sexual dysfunction. The Female Sexual Function Index (FSFI) questionnaire analyzes six domains of sexual function: desire, arousal, lubrication, orgasm, satisfaction and pain, and emphasizes arousal disorder, with $>27.5$ being a good sexual function. The overall mean score was 28.30 , showing good sexual function and in the individual average, $28 \%$ of the participants suffered from sexual dysfunction. It was concluded that the university women in this study had good sexual satisfaction, however, with a high percentage of sexual dysfunction analyzed by the FSFI questionnaire.

Keywords: sexual satisfaction, sexual dysfunction, FSFI questionnaire, physiotherapy.

\section{INTRODUÇÃO}

A Organização Mundial de Saúde (OMS) em 1983 estabeleceu que a resposta do ciclo feminino possui quatro fases (desejo, excitação, orgasmo e resolução), esta versão do modelo linear do ciclo de resposta sexual feminina tem sido utilizada até os dias atuais ${ }^{1-3}$. A disfunção sexual é qualquer alteração, em qualquer das quatro fases do ciclo de resposta sexual. De acordo com a OMS, a disfunção sexual enquadrase na classificação internacional de doenças como uma incapacidade que tenha uma duração mínima de seis meses ${ }^{4,5}$.

A disfunção sexual tem multicausalidade como fator etiológico, onde envolve não só o biológico, mas sim o indivíduo biopsicossocialmente, onde a interação do ser humano como um todo gera papel primordial em suas respostas biológicas, culturais, sociais e psicológicas. Tudo que ocorre na vida da mulher pode repercutir em respostas inibitórias da libido, como por exemplo, a pubalgia, endometriose, 
anorgasmia, vaginismo, dor pélvica crônica, tumores ou até mesmo alterações anatômicas congênitas, estresse, religiosidade, disfunção sexual do parceiro, depressão, baixa autoestima com relação a sua imagem corporal, relapso sexual, abuso e o uso constante de alguns fármacos que suprimem a resposta sexual ${ }^{6-10}$.

Nos dias atuais a disfunção sexual tem aumentado muito entre as mulheres jovens, porém este tema é pouco abordado, seja na saúde pública ou privada e/ou instituições de ensino, pois ainda existe um tabu quando o assunto é sexualidade, principalmente feminina, o que gera receio em abordar este assunto. Outro agravante que muito limita a intervenção precoce nas disfunções sexuais é a falta de informação ou as que têm conhecimento possuem vergonha de falar com o médico, fisioterapeuta e parceiros sobre o problema e quando procuram ajuda acabam desistindo da intervenção por medo de não ser efetiva ${ }^{4,10-12}$

Ribeiro, afirma em seu estudo que a prevalência na horizontalidade mundial, da disfunção sexual é de $25-63 \%$, porém relata que há uma carência de estudos nesta área, afirmando também, que os estudos realizados são híbridos, ou seja, utilizam diversos métodos para sua mensuração e que necessita de estudos com metodologias consistentes para obtenção de resultados mais precisos ${ }^{4}$.

Assim, dentro da gama de instrumentos avaliativos para os aspectos de disfunção sexual, destaca-se o questionário Female Sexual Function Index (FSFI), por ser fácil de administrar e analisar, na qual, foi avaliado e transcrito para a língua portuguesa, de maneira firme e seguindo fielmente os parâmetros metodológicos do questionário, validando a sua fidedignidade nos resultados para que sua aplicabilidade fosse a mesma do que a do questionário original ${ }^{13}$.

O FSFI foi concebido por um grupo multidisciplinar de especialistas da disfunção sexual feminina ${ }^{15}$. Suas esferas e subitens foram baseados na classificação de disfunção sexual feminina da American Foundation for Urologic Disease (AFUD). São 19 itens que analisam seis domínios da função sexual: desejo, excitação, lubrificação, orgasmo, satisfação e dor, enfatizando o distúrbio da excitação. Esta categoria é subdividida em dois domínios separados de lubrificação (quatro itens) e excitação propriamente dita (quatro itens), permitindo avaliar componentes periféricos (lubrificação) bem como centrais (excitação subjetiva e desejo) ${ }^{13}$.

Diante do apresentado, este trabalho é de suma importância para que haja mais atenção ao público feminino jovem, que também sofre com disfunção sexual, uma vez que, é escasso na literatura estudos que abordem tal temática nesta faixa etária, assim, esse público fica susceptível a nunca saber se sofre de alguma disfunção ou que há tratamento. Muito se tem estudado sobre a satisfação sexual em mulheres no climatério, gestantes e com patologias, entretanto, pouco se investe em pesquisas para o público feminino jovem. Assim, o objetivo do estudo foi avaliar a satisfação sexual em mulheres universitária.

\section{MÉTODOS}

Estudo transversal, aprovado pelo Comitê de Ética em Pesquisa, protocolo no CAAE 55875116.0.0000.5512. A amostra foi composta por 100 universitárias, com idade entre 18 e 35 anos, com vida sexual ativa, estudantes do período noturno de uma universidade privada em Assis-SP e que não realizaram tratamento de disfunção sexual nos últimos 3 meses.

As participantes foram abordadas para receberam explicações sobre o objetivo e procedimentos da pesquisa, assim como verificar o interesse em participar do estudo. Após concordarem com a aplicação do questionário, assinaram o termo de consentimento livre e esclarecido e em seguida, receberam o questionário Female Sexual Function Index (FSFI) para ser respondido sem qualquer tipo de ajuda e de forma individual.

O questionário é composto por uma escala numérica capaz de avaliar cada domínio separadamente ou todo arranjo. Nas questões 3 a 14 e 17 a 19, a escala varia de $0-5$ e nas questões 1, 2, 15 e 16, de 1-5. O resultado global é definido pela totalidade de cada domínio multiplicado por seu fator correspondente e pode variar entre 2 a 36 . O ponto de corte para uma boa função sexual é $>27,5$; como demonstrado durante o processo de validação deste instrumento numa população de mulheres entre 18-74 anos com e sem disfunção sexual feminina $(D S F)^{13-16}$. A pontuação total do FSFI se deve a soma de todas as pontuações de todas as dimensões, os valores variam entre 0 a $6 / 1,2$ a 6 o valor total deste questionário é de 2-36, onde quanto menor a pontuação pior é a satisfação 
sexual e pode ser correlacionada à alguma disfunção sexual ${ }^{1}$.

A análise do questionário foi feita com base na Tabela 1, sendo multiplicado o valor do domínio por seu fator correspondente, e para média geral foi realizado a soma de todos os domínios já multiplicados posteriormente por seu fator equivalente ${ }^{13-15}$.

Tabela 1. Escores dos domínios do Female Sexual Function Index.

\begin{tabular}{lccccc}
\hline Domínio & Questão & Variação do escore & Fator & Escore mínimo & Escore máximo \\
\hline Desejo & 1,2 & $1-5$ & 0,6 & 1,2 & 6,0 \\
Excitação & $3,4,5,6$ & $0-5$ & 0,3 & 0 & 6,0 \\
Lubrificação & $7,8,9,10$ & $0-5$ & 0,3 & 0 & 6,0 \\
Orgasmo & $11,12,13$ & $1-5$ & 0,4 & 0 & 6,0 \\
Satisfação & $14,15,16$ & 0 (ou 1) - 5 & 0,4 & 0,8 & 6,0 \\
Dor & $17,18,19$ & $0-5$ & 0,4 & 0 & 6,0 \\
Escore Total & & & & 2,00 & 36,00 \\
\hline
\end{tabular}

\section{RESULTADOS}

Analisando os questionários, observou-se que o escore geral das universitárias foi em média de 28,30 pontos, o que significa boa satisfação sexual. Embora o valor seja baixo, considerando que o questionário vai de 2-36 pontos, mesmo as participantes apresentando-se satisfeitas, observou-se que elas têm dificuldades para atingir o orgasmo (Tabela 2).

Tabela 2. Análise do escore do questionário Female Sexual Function Index em universitárias.

\begin{tabular}{lccccccc}
\hline FSFI & Desejo & Excitação & Lubrificação & Orgasmo & Satisfação & Dor & Escore geral \\
\hline Média & 4,42 & 4,38 & 4,89 & 4,51 & 5,34 & 4,74 & 28,30 \\
Ponto Mínimo & 1,20 & 0 & 0 & 0 & 0 & 0 & 3,60 \\
Ponto Máximo & 6,00 & 6,00 & 6,00 & 6,00 & 6,00 & 6,00 & 34,20 \\
10 quartil & 3,60 & 3,90 & 4,50 & 4,10 & 5,20 & 4,40 & 27,12 \\
2o quartil & 4,80 & 4,50 & 5,10 & 4,80 & 6 & 5,20 & 30,15 \\
3o quartil & 5,40 & 5,32 & 5,70 & 5,60 & 6 & 5,90 & 31,70 \\
DP & 1,09 & 1,15 & 1,23 & 1,32 & 1,17 & 1,57 & 5,70 \\
\hline
\end{tabular}

Nota: DP= Desvio Padrão

Quanto à pontuação do escore geral de cada participante, $28 \%$ das universitárias apresentaram pontuação $<27,5$, sendo indicativo para disfunção sexual (Tabela 3). Um dos domínios que foram identificados como mais acometidos foram o domínio do "desejo", demonstrando baixo interesse sexual; "excitação", com dificuldade em sentir-se e/ou manter-se excitada durante a relação sexual, o que consequentemente leva a dificuldades para chegar ao orgasmo (Figura 1). 
Tabela 3. Análise da pontuação do escore geral, por participante, do questionário Female Sexual Function Index (FSFI).

\begin{tabular}{lcccc}
\hline Pontuação FSFI & \multicolumn{2}{c}{$<\mathbf{2 7 , 5}$} & \multicolumn{2}{c}{$>\mathbf{2 7 , 5}$} \\
\hline Participantes & $\mathrm{N}$ & $\%$ & $\mathrm{~N}$ & $\%$ \\
\hline 100 & 28 & 28 & 72 & 72 \\
\hline
\end{tabular}

Média dos domínios da satisfação sexual $(n=100)$

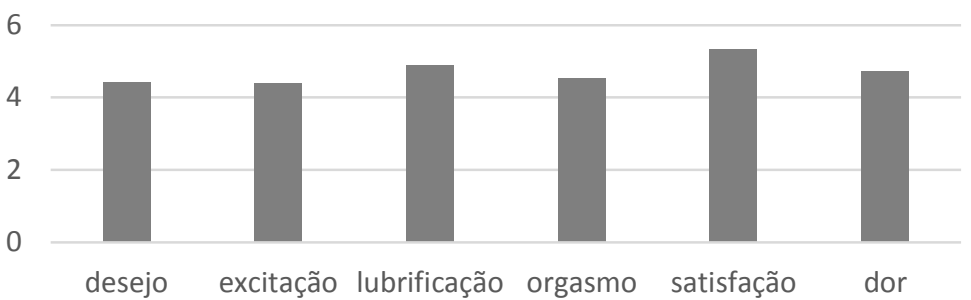

Figura 1. Média dos domínios da satisfação sexual analisado pelo Female Sexual Function Index.

\section{DISCUSSÃO}

Segundo a análise dos questionários no presente estudo cerca de $30 \%$ das universitárias com idade entre 18 a 35 anos sofrem de disfunção sexual, na qual, esse dado entra em consonância com outro estudo que verificou a qualidade sexual de mulheres e observou que $40 \%$ das mulheres com idade entre 18 a 59 anos sofrem de alguma disfunção sexual ${ }^{16}$.

Ainda foi possível verificar que as participantes apresentaram baixo desejo/ interesse sexual e dificuldades para se sentirem excitadas assim como para chegar ao orgasmo/clímax, o que se soma com um estudo que evidenciou uma elevada porcentagem de mulheres com transtorno orgástico ou transtorno de desejo, correspondendo, em ambos os casos $35 \%^{8}$.

Ribeiro e colaboradores realizaram uma pesquisa em uma população de mulheres com idade entre 18-58 anos e evidenciou prevalência da DSF de $77,2 \%$, tendo o distúrbio orgástico o mais prevalente com $55,8 \%{ }^{4}$. Já em outro estudo, as principais queixas femininas foram a falta de desejo sexual compreendendo $34,6 \%$ da amostra e a dificuldade de orgasmo para $29,3 \%$ das participantes, além disso, mostrou que esse índice aumenta com a idade ${ }^{17}$.

No estudo encabeçado por Fonseca verificou que $40,9 \%$ das estudantes avaliadas referiram que na maioria das vezes atingiam o orgasmo nas relações sexuais que realizavam, além de possuírem bom desempenho sexual e que sentiam desejo a maior parte do tempo, sendo achados que diferem dos resultados encontrados no presente estudo ${ }^{18}$.

O domínio mais acometido no presente estudo foi o "desejo sexual", muitas vezes por não serem estimuladas suficientemente por seus parceiros, tendo dificuldades para ficarem e/ou se sentirem excitadas, não conseguem manter a lubrificação durante toda a relação sexual, e tais alterações dificultam ou impedem o orgasmo, gerando um ciclo de resposta sexual feminina incompleto, que causa desconforto a maior parte do tempo e pode levar a diminuição do interesse sexual das universitárias, além de fazer com que as jovens se sintam frustradas e tenham experiências sexuais negativas por não conseguirem se satisfizer sexualmente.

Apesar da contribuição deste estudo, ainda assim apresenta algumas limitações, nas quais se referem à amostra que poderia abranger mais participantes, idades diferentes, diferentes percepções da satisfação sexual e que podem ter influenciado no resultado final, logo, futuros estudos deverão considerar esses apontamentos para apresentação de resultados mais precisos.

Conclui-se que as mulheres universitárias, entre 18 e 35 anos de idade apresentam boa satisfação sexual analisada pelo questionário FSFI demonstrado pelo escore geral em média de 
28,30 pontos, porém, $28 \%$ das participantes apresentaram alguma disfunção sexual. Em conjunto, sugere-se que novos estudos sejam realizados para que as mulheres sejam conscientizadas e que enfrentem o tabu da sexualidade e assim procurar ajuda profissional para a resolução das disfunções sexuais.

\section{CONFLITO DE INTERESSE}

Os autores declaram não haver qualquer potencial conflito de interesse que possa interferir na imparcialidade deste trabalho científico.

\section{REFERÊNCIAS}

1. Pechorro P, Diniz A, Almeida S, Vieira R. Validação portuguesa do índice de Funcionamento Sexual Feminino (FSFI). Labor Psicol. 2009;7(1):33-44.

2. Pechorro P, Diniz A, Vieira R. Satisfação sexual feminina: Relação com funcionamento sexual e comportamentos sexuais. Análise Psicol. 2009;27(1):99-108.

3. Bedone RM Volpato Resposta sexual, disfunção sexual e qualidade de vida em mulheres obesas BIBLIOTECA DIGITAL DA USP. [Tese de doutorado]. São Paulo: Univesidade de São Paulo; 2014.

4. Ribeiro B, Magalhães AT, Mota I. Disfunção sexual feminina em idade reprodutivaprevalência e fatores associados. Rev Port Med Geral Fam. 2013;29(1):16-24.

5. Abdo CHN. Quociente sexual feminino:um questionário brasileiro para avaliar a atividade sexual da mulher. Diagn Tratamento. 2009;14(2):89-1.

6. Faubion SS, Rullo JE. Disfunção sexual na mulher:uma abordagem prática. Rev Port Med Geral Fam. 2015;35(1-3):281-8.

7. Taquette SR, e Vilhena MM. Uma contribuição ao entendimento da iniciação sexual feminina na adolescência. Psicol Estudo. 2008;13(1):105-114. DOI: $\quad$ http://dx.doi.org/10.1590/S1413$\underline{73722008000100013}$

8. Piassarolli VP, Hard E, Andrade NF, Ferreira NO, Osis MJD. Treinamento dos músculos do assoalho pélvico nas disfunções sexuais femininas. Rev Bras Ginecol Obstet. 2010;32(5):334-340. DOI: http://dx.doi.org/10.1590/S0100-

\section{6}

9. Aveiro MC, Garcia APU, Driusso P. Efetividade de intervenções fisioterapêuticas para o vaginismo: uma revisão da literatura. Fisioter Pesq. 2009;16(3):279-83. DOI: http://dx.doi.org/10.1590/S180929502009000300016

10.Vilarinho SMDC Serapicos Funcionamento e satisfação sexual feminina:Integração do afecto, variáveis cognitivas e relacionais, aspectos biológicos e contextuais. [Tese de doutorado]. Coimbra: Univesidade de Coimbra; 2007.

11. Lara LAS, Ana CJSRS, Romão APMS, Junqueira FRR. Abordagem das disfunções sexuais femininas. 2008;30(6):312-21. DOI: http://dx.doi.org/10.1590/S010072032008000600008

12. Abdo CHN, Fleury HJ. Aspectos diagnósticos e terapêuticos das disfunções sexuais femininas. Rev Psiq Clín. 2006;33(3):162-167. DOI: http://dx.doi.org/10.1590/S0101$\underline{60832006000300006}$

13. Pacagnella RC, Martinez EZ, Vieira EM, Validade de construto de uma versão em português do Female Sexual Function Index. Cad Saúde Púb. 2009;25(11):2333-2344. DOI: http://dx.doi.org/10.1590/S0102$\underline{311 \times 2009001100004}$

14. Pacagnella RC, Vieira EM, Oswaldo MRJ, Souza C. Adaptação transcultural do Female Sexual Function Index. Cad Saúde Pública. 2008;24(2):416-426. DOI: http://dx.doi.org/10.1590/S0102311X2008000200021

15. Hentschel H, Alberton DL, Capp E, Goldim JR, Passos EP. Validação do female sexual function index (FSFI) para uso em língua portuguesa. Rev. HCPA. 2007;27(1):10-14.

16. Blümel MJE, Binfa LE, Cataldo PA, Carrasco AL, Izaguirre HL, Sarrá SC. Índice de función sexual femenina: un test para evaluar la sexualidad de la mujer. Rev Chil Obstet Ginecol. 2004;69(2):118- 
125. DOI: http://dx.doi.org/10.4067/S0717$\underline{75262004000200006}$

17. Abdo CHN, Oliveira JWM, Moreira ED, Fittipaldi JAS, Perfil sexual da população brasileira: resultados do Estudo do Comportamento Sexual (ECOS) do Brasileiro. Rev Bras Med. 2002;59:250-257.

18. Fonseca MFSM, Beresin R. Avaliação da função sexual de estudantes de graduação em Enfermagem. 0 Mundo da Saúde. 2008;32(4):430-436.

Recebido para publicação em 09/08/2018

Revisado em 24/08/2018

Aceito em 11/09/2018 